\title{
New Targets and Drugs in Cancer Chemotherapy
}

\author{
Thomas Szekeres $^{\mathrm{a}}$ Ladislav Novotny $^{\mathrm{b}}$ \\ aClinical Institute of Medical and Chemical Laboratory Diagnostics, University of Vienna Medical School, \\ General Hospital of Vienna, Austria; bDepartment of Pharmaceutical Chemistry, Faculty of Pharmacy, \\ Kuwait University, Kuwait
}

\section{Key Words}

Cancer chemotherapy · Apoptosis · Drug targets . Antitumor strategies

\begin{abstract}
Cancer is still the number two cause of death in highly developed countries. Due to increased life expectancy, the number of cancer cases has also increased, and it is not likely that the incidence of this often fatal illness will decrease in the near future. Breast cancer, for instance, strikes 1 of 8 women in the industrialized world. Incidence of lung cancer increases due to smoking, while that of colon cancer increases with age. These are only a few examples highlighting the growing importance of strategies effective for cancer treatment. Despite surgical treatment and irradiation, chemotherapy remains one of the important means to treat cancer. Although many institutions and companies have tried to establish new treatment strategies such as immunotherapy or vaccination, the conventional chemotherapy still remains an important treatment option. In this review, we present an overview of new and innovative treatment strategies for cancer chemotherapy. A number of new targets as well as anticancer compounds are introduced and though we are well aware of the fact that the list is incomplete, we hope to be able to provide insight into this rapidly developing field.
\end{abstract}

Copyright @ 2002 S. Karger AG, Basel

\section{Introduction}

Despite the use of surgical treatment and irradiation, chemotherapy still remains an important option for the treatment of various malignancies. The most important obstacle for the successful chemotherapeutic treatment remains the identification of a suitable target molecule or enzyme. The chemotherapeutic drug should preferentially target tumor cells without harming normal cells or tissues. Unfortunately, in most cases, chemotherapy remains a treatment modality with severe side effects due to the toxicity of the drugs. Bone marrow depletion and toxic effects on rapidly proliferating cells such as intestinal cells can cause life-threatening infections and side effects, which seriously limit the quality of life of the patient. Therefore the ideal goal of an effective chemotherapy is to identify targets and molecules that specifically attack tumor cells and cause only minor harm to healthy organs and cells.

Various researchers have committed themselves to this task. Many biochemical pathways have been investigated to find significant differences between tumor cells and normal healthy cells in order to identify suitable targets for anticancer therapy. An overview of potential targets that are overexpressed in a transformation- and proliferation-linked manner are known. Therefore, in this review, we focus on a number of selected targets and their inhibitors, such as DNA methylation, caspases, protein kinase or ribonucleotide reductase. These targets can be modulated by drugs which are widely used in cancer chemother-

Thomas Szekeres, MD

Clinical Institute of Medical and Chemical Laboratory Diagnostics

University of Vienna Medical School, General Hospital of Vienna

Währinger Gürtel 18-20, A-1090 Vienna (Austria)

Tel.+43140400 5365, Fax +43140400 5390,E-Mail thomas.szekeres@univie.ac.at 
apy as well as antiviral chemotherapy. In addition, several newer compounds are being synthesized, all targeting enzymes or pathways that are overexpressed in tumor cells and have only limited importance in resting nontumorous tissue. Therefore, inhibiting these enzymes or pathways might cause malignant cells to undergo apoptosis or necrosis with relatively limited side effects.

\section{DNA Methylation}

Szyf [1] pointed out that understanding new gene expression programs permits pharmacological intervention, as processes controlling genetic programs can become new drug targets. DNA methylation, for instance, plays an important role in the control of genetic expression; therefore, alteration of their function might result in therapeutic effects [1].

Indeed, DNA methylation is an important mechanism of DNA modification resulting in altered gene expression as described by Karpf et al. [2]. Essentially, inhibition of tumor suppressor genes by DNA methylation was described in cancer cell lines and in human tumors. Therefore, the enzyme DNA methyltransferase has become an important drug target. 5-Aza-2'-deoxycytidine [5-azaCdR (decitabine)], a potent inhibitor of DNA methyltransferase, is a drug now in clinical trials for the treatment of solid tumors and leukemia. The efficacy of 5-aza$\mathrm{CdR}$ may be related to genomic hypomethylation, and/or enzyme-DNA adduct formation. 5-Aza-CdR treatment activates the tumor suppressor p53. Karpf et al. [2] showed that colon tumor cell lines expressing wild-type p53 are more sensitive to 5-aza-CdR. This treatment includes the induction and activation of wild-type but not mutant p53 protein. The induction of p53 protein after 5-aza-CdR treatment did not correlate with an increase in p53 transcripts. This indicates that hypomethylation at the p53 promoter does not account for the p53 response. 5-Aza-CdR has shown promising results in clinical trials for the treatment of chronic myelogenous leukemia [2]. A recent study reveals that the pattern of $\mathrm{CpG}$ island methylation is characteristic of tumor type, suggesting that distinct sets of genes are inactivated by methylation during development of each tumor type. Several loci were in fact identified as being frequently methylated in liver tumors [3]. For example treatment of tumor cell lines with 5-aza$\mathrm{CdR}$ was reported to result in an expression of mlt 1 gene, indicating that the repression of mlt 1 is connected to methylation. Mlt 1 is a target gene that is silenced by methylation in liver tumors.
Recently Yang et al. [4] have demonstrated that DNA methylation and transcriptionally inactive chromatin are related. Because the absence of estrogen receptor alpha (ERalpha) gene expression has been associated with aberrant methylation of its $\mathrm{CpG}$ island in breast cancers, Yang et al. [4] investigated whether histone deacetylase activity contributes to the transcriptional inactivation of the methylated ER gene. This was tested in various ER-negative human breast cancer cells. Treatment of these cells with trichostatin A, a specific histone deacetylase inhibitor, led to dose- and time-dependent reexpression of ER mRNA as detected by reverse transcription-PCR without alteration in ERalpha $\mathrm{CpG}$ island methylation. Trichostatin A-induced expression was associated with increased sensitivity to DNAse I at the ER locus in MDA-MB-231 cells. These data indicate that inactive chromatin, which is mediated by histone deacetylation, is a critical component of ER gene silencing in human breast cancer cells. Therefore, histone deacetylation represents a target for treatment of certain ER-negative breast cancers [4].

\section{Caspade Proteinases - Caspases}

Many reports have been published clarifying the role and effects of caspases in tumor cells [5-11]. In this review, we include a few examples to illustrate their therapeutic potential, which is directly related to apoptotic death event in malignant cells. Some caspase inhibitors have already been tested in various cell lines [5]. The caspase inhibitor z-VAD.fmk, for instance, provides protection against apoptosis mediated by some specific peptides by blocking the dimerization through which Bcl-2 would normally inhibit apoptosis which opens other apoptotic pathways.

Kim et al. [6] investigated transforming growth factor$\beta_{1}$ (TGF- $\left.\beta_{1}\right)$, a growth inhibitor, for its apoptotic effect. They showed that the mechanism of TGF- $\beta_{1}$-induced apoptosis in SNU-16 human gastric cancer cells was related to their initial arrest at the $G_{1}$ phase accompanied by the caspase- 3 activation. Consequently, cells proceeded into apoptosis [6]. These results suggest that activation of caspase- 3 by TGF- $\beta_{1}$ may initiate the conversion from $\mathrm{G}_{1}$ cell cycle arrest to apoptosis via the cleavage of p21, p27 and $\mathrm{Rb}$, which in turn causes $\mathrm{Cdk} 2$ activation. $\mathrm{Cdk} 2$ activation itself is a downstream effector of caspase and is a critical step for the execution of TGF- $\beta_{1}$-induced apoptosis [6].

Cellular sensitivity to therapeutics cannot be readily enhanced by aiming for just one target in the apoptotic 
pathway as other cell elements might carry on the functions of a former target [7]. In addition, even elimination of a whole pathway may have little effect on sensitivity because cellular viability is protected by so many different mechanisms. Nevertheless, where molecular changes have a phenotypic consequence, development of novel therapeutic strategies might be possible. One example is a recently identified organic compound that can inhibit p53 function and thus protect normal tissues against radiation-induced apoptosis.

Novel cyclic hydroxamic acid derivatives have demonstrated varying degrees of inhibitory effects on the arachidonate 12-lipoxygenase. Therefore, their effect against human prostate cancer cells has been studied, confirming the apoptotic effect of caspases by the activation of caspase- 3 from procaspase- 3 by caspase- 9 [8]. The data presented in $\mathrm{Li}$ et al. [9] suggest that these novel cyclic hydroxamic acid derivatives inducing apoptosis may find potential clinical applications in cancer chemotherapy.

It is known that treatment of HL-60 human leukemia cells with etoposide induces apoptotic cell death. In a study by Martins et al. [10] simultaneous activation of at least 18 electrophoretically distinct cysteine-dependent aspartate-directed protease (caspase) isoforms were detected. They investigated whether activated caspases are phosphorylated, as phosphorylation of active caspases could have an inhibitory effect on enzyme activity, and reported that as caspases are phosphoproteins, they may serve as possible targets for protein kinases that are involved in apoptotic events [10].

Tse and Rabitts [11] have reported on the use of intracellular antibodies for cancer therapy via apoptotic cell death. Apparently apoptosis is triggered by specific antibody-antigen interaction by intracellular antibodies linked to caspase-3, which induces cell killing. They found in vivo coexpression of an antibody-caspase- 3 fusion with its antigenic target, induced apoptsis specific for antibody-antigen, and active caspase-3. Moreover, the antibody-caspase- 3 fusion protein was not toxic to cells in the absence of antigen. Therefore, they concluded that intracellular antibody-mediated apoptosis should be useful as a specific therapeutic approach for the treatment of cancers [11].

\section{Various Protein Kinases}

Protein kinases play a crucial role in signal transduction as well as in cellular proliferation, differentiation, and various regulatory mechanisms. Therefore, the inhi- bition of growth-related kinases, especially tyrosine kinases, might provide new therapies for diseases such as cancer. For example, recent evidence suggests that the enzyme serine/threonine kinase is involved in the divergent cellular responses of some cell lines [12]. The progress made in the crystallization of protein kinases has confirmed that the ATP-binding domain of tyrosine kinases is an attractive target for drug design. One example of drug design using a tyrosine kinase as target is a pyrrol (2, 3-d)pyrimidine derivative, which acts as a dual inhibitor of both the epidermal growth factor receptor (EGFR) and the ErbB2 kinases. The compound underwent clinical trials in 1999, based on its favorable preclinical profile: potent inhibition of epidermal growth factor (EGF)mediated signaling in cells, in vivo antitumor activity in several EGFR overexpressing xenograft tumor models in nude mice, long-lasting inhibition of EGF-stimulated EGFR autophosphorylation in tumor tissue, good oral bioavailability in animals, and no prohibitive in vitro and in vivo toxicity findings [13]. The anilinophthalazine derivative PTK787/ZK222584 is a potent and selective inhibitor of both KDR and Flt-1 kinases with interesting antiangiogenic and pharmacokinetic properties. STI571, a phenylamino-pyrimidine derivative, is a potent inhibitor of the Abl tyrosine kinase, which is present in $95 \%$ of patients with chronic myelogenous leukemia (CML). The compound specifically inhibits proliferation of $\mathrm{v}-\mathrm{Abl}$ - and Bcr-Abl-expressing cells (including cells from CML patients) and shows antitumor activity as a single agent in animal models at well-tolerated doses. Pharmacologically relevant concentrations are achieved in the plasma of animals (oral administration). Promising data from phase I and II clinical trials in CML patients (98\% hematological response rate in phase I) support the fact that STI571 represents a new and successful treatment modality for CML. In addition, potent inhibition of the PDGFR and c-Kit tyrosine kinases also indicates its possible clinical use in solid tumors [14].

The group of kinases includes also extracellular signals known as growth factors, which control cell growth and differentiation. Growth factors are high-affinity ligands for transmembrane receptors belonging to the family of receptor tyrosine kinases (RTKs). Longati et al. [15] described the role of RTKs in human diseases, such as developmental disorders or cancer. Mutations involving the Ret receptor were found in patients suffering from multiple endorine neoplasia or familial thyroid carcinomas. Mutations in the Kit receptor have been found to be connected to mastocytomas and gastrointestinal tumors. Germline and sporadic mutations of the Met receptor 
have been described in kidney and hepatocellular carcinomas. In addition overexpression of the HER-2/neu receptor in breast cancer has been associated with tumor progression. HER2/neu is therefore considered to be a target for cancer chemotherapy [15].

\section{Metalloproteinases}

The proteolytic processes are thought to be important in tumor invasion and metastasis, mainly by matrix metalloproteinases (MMPs) and serine proteases. The differential expressions of MMP-2 activation and production during the different stages of breast cancer progression are potential therapeutic targets for biological or gene therapy [16].

Treatment of ovarian cancer cells, NOM1, with fibronectin (FN) stimulates matrix metalloproteinases-9 (MMP-9) secretion and thus activates the invasiveness of cells via the FAK/Ras signaling pathway. Traxler et al. [13] found that the downstream effector, which is critical for FN-dependent secretion of MMP-9, was inhibited and that treatment of cells with these inhibitors dramatically suppressed the secretion of MMP-9. Similarly, P1-3 kinase inhibitors strongly suppressed the FN-dependent secretion of MMP-9. In contrast, a specific PKC inhibitor (GF109203X) showed no inhibitory effect on the FNdependent MMP-9 secretion. Moreover, it was reported that both the MEK1 inhibitor and the P13-K inhibitor, but not the PKC inhibitor, strongly suppressed the invasiveness of tumor cells. These results suggest that activation of dual signaling pathways is required for the FNdependent activation of MMP-9 secretion, indicating the importance of these signaling molecules as chemotherapeutic targets for cancer [13].

\section{Topoisomerases I and II}

Camptothecins are topoisomerase I inhibitors. Topoisomerases play different roles during apoptosis when compared to cell cycle arrest events [17]. This behavior makes them interesting from the point of view of cancer chemotherapy. Because of the complexity of apoptotic pathways, the importance of multifactorial analyses for modulating/predicting the apoptotic response to chemotherapy has to be considered.

The topoisomerase inhibitors have become a part of second-line therapeutic regimens for small-cell lung cancer (SCLC) and non-small-cell lung cancer (NSCLC) [18].
They are alternatives to several promising new agents like taxanes, mitotic spindle inhibitors or antimetabolites. Therefore, topoisomerases can serve as molecular targets for these new substances. Their design is based on the fact that topoisomerase I, an enzyme that is essential for DNA replication, is up-regulated in tumor cells. Inhibition of this enzyme by drugs such as topotecan and irinotecan leads to cell death, which is the basis for their anticancer activity. The process of DNA replication is halted by the covalent binding of the drug in a topoisomerase I drug/ DNA ternary reaction intermediate. The pharmacokinetics of the approved regimen (a 30-min infusion daily for 5 days at 21-day intervals) is well defined and the antitumor activities of both the intravenous and oral formulation of topotecan have been extensively tested in clinical trials [18]. Topotecan is well tolerated and has demonstrated good efficacy in patients with relapsed SCLC when administered as monotherapy or in combination regimens. Preliminary trials also indicate that topotecan is well tolerated and has activity in the first-line treatment of NSCLC. It also became the first topoisomerase I inhibitor to be approved for second-line therapy in SCLC. Other studies and trials with these compounds indicate promising results for therapy of SCLC and NSCLC.

\section{Farnesyl Transferase}

Inhibitors of the enzyme farnesyl protein transferase prevent a key step in the posttranslational processing of the Ras protein and were thus initially developed to inhibit cell signaling in Ras-transformed cells. Due to the biological effects of farnesyl transferase inhibitors (FTIs) on cancer cells, tumors without oncogenic Ras may also be targets for FTI therapy [19, 20]. The development of FTIs was based on the fact that proto-oncogenes, which cause overexpression or aberrant function of their encoded protein, represent a target for novel therapeutic substances. The introduction into clinical practice of trastuzumab as a monoclonal antibody targeted against breast carcinomas overexpressing the growth factor receptor HER-2/neu provides the first validation of this approach [21].

There are three Ras proto-oncogenes (H-, N- and KRas) encoding a total of four 21-kD Proteins (H-Ras, NRas, K-Ras 4A and K-Ras 4B) [22]. They transmit a variety of extracellular signals from the cell surface, including growth factors, cytokines and hormones [23].

The farnesylation transferring a farnesyl isoprenoid group from farnesyl diphosphate and forming a thioether bond with the cysteine moiety in the C-terminal tetrapep- 
tide sequence of the Ras protein is essential for the Ras protein to function in the signal transduction cascade [19]. Indeed, the elucidation of this process resulted in the first attempts of rational drug design based on the preparation of peptidomimetic compounds simulating the Cterminal tetrapeptide sequence of Ras [24, 25]. New prodrugs were found to inhibit the growth of more than $70 \%$ of tumor cell lines tested [26]. Moreover, as FTIs became a center of attention, new compounds were synthesized. Compounds which are active in the nanomolar range, i.e. FTIs based on the benzophenone core structure, are presently being tested [20].

Several FTIs have entered clinical development [19]. In the first phase II study of an FTI in breast cancer, patients were treated with R1 15777 and it was shown [27] that inhibition of signal transduction by FTIs was therapeutically effective [19]. Additionally, R115777 was already used in poor-risk acute leukemia patients [28] and in patients with advanced solid tumors with no standard therapy available [29]. The results suggest that the use of FTIs may have important clinical implications [30].

\section{Telomerase}

Lavelle et al. [31] recently reviewed telomerase as a therapeutic target for the control of cell proliferation. It acts by interfering with cell senescence. Telomerase might become a therapeutic target due to the correlation between the level and frequency of telomerase expression and the malignant properties of tumors. This correlation has now been shown in established tumor cell lines and fresh tumor samples. Telomerase expression significantly differs between malignant and normal tissues. This difference is more significant than the differences observed in enzymatic targets of chemotherapy such as thymidylate synthetase, dihydrofolate reductase and topoisomerases. Therefore, telomerase inhibitor might show a good selectivity for tumor cells with minimal toxicity for normal tissues. According to the paradigm currently proposed for telomeres and telomerases, it can be predicted that telomerase inhibition will not affect a tumor until its telomeres reach the critical size for entering senescence. This means that during antitelomerase therapy, the tumor cells will continue to grow and undergo 20-30 divisions until the telomeres reach a critical size leading to tumor senescence.

This understanding might be important in patients with advanced tumors at the beginning of therapy. Ultimately, the definitive solution of this problem will come from elucidating the properties of telomerase inhibitors. Different strategies will be used for testing new telomerase inhibitors. These new agents, telomerase inhibitors, will include direct inhibitors of telomerase activity, substances interfering with telomeres and compounds interacting with other proteins involved in the regulation of telomerase and telomeres [31].

The effects of a candidate telomerase inhibitor, penclomedine, in glioma cells have already been documented [32] in human malignant glioma cell lines. All of them were telomerase-positive and their telomerase activity was not modulated during cell cycle progression, did not correlate with p53 status or bcl-2 family protein expression, and did not predict drug sensitivity, except for an association with resistance to carmustine. Additionally, ectopic bcl-2 expression did not enhance telomerase activity. Wild-type p53 reduced telomerase activity in cell lines retaining p53 activity but not in p53-mutant cell lines. Penclomedine killed glioma cells via an apoptotic, but bcl-2- and caspase-independent pathway. However, it did not inhibit telomerase and did not act synergistically with cytotoxic drugs. Consequently, it was concluded that telomerase activity does not account for the differential chemosensitivity of human glioma cells and that penclomedine kills glioma cells via a telomerase-independent pathway [32].

\section{Prolactin}

The mammary gland is the major target tissue of prolactin (PRL) in mammals. Although this pituitary hormone has been suspected to be involved in the progression of human breast cancer, the failure of clinical improvement by treatment with dopamine agonists (which lower circulating levels of PRL) rapidly reduced the interest of oncologists concerning a potential role of PRL in the development of breast cancer [33]. Over the last few years, however, several studies reported that mammary epithelial cells also synthesize PRL and that prolactin may have a proliferative activity in an autocrine/paracrine manner. These observations have led to a reconsideration of the role of PRL as a therapeutic target in breast cancer.

In order to define new molecular targets, the PRL receptor signaling cascades known to be triggered by PRL in mammary epithelial cells were proposed as potential antitumor targets [33], suggesting that human PRL could also be a molecular target in the search for strategies in the treatment of breast cancer [34]. 


\section{Cell Surface Antigens}

Tumor toxins are recombinant polypeptides derived from the fusion of a single-chain antibody domain or a ligand specific for a cell surface receptor with the enzymatic domain of a bacterial or plant toxin. If these receptors are preferentially expressed on the surface of tumor cells, the tumor toxins can serve as efficient therapeutics. Examples in which growth factors, cytokines, hormones or antibody domains were fused to toxins have been described by Wick and Groner [35]. These bacterially expressed molecules have shown antitumor effects in vitro and in vivo. Since many tumors are not sensitive to existing tumor toxins, the search continues for new antigens with tumor-specific or tumor-enhanced expression. In a study by Wick and Groner [35] three antigenic structures were explored for their possible use as targets for tumor toxins, i.e. (a) a glycosphingolipid with an Le(a) antigenic structure that is overexpressed in gastrointestinal carcinomas, e.g. colon carcinoma, (b) the epithelial glycoprotein episialin, which shows altered expression in various tumor tissues such as breast, ovary and pancreas, and (c) the neurotensin receptor that is found on NSLC, pancreatic and colonic cancer cells. For attaching the tumor toxin to the carbohydrate structures, recombinant single-chain antibody domains ( $\mathrm{scFv}$ ) fused to a truncated form of the exotoxin A (ETA) from Pseudomonas aeruginosa were constructed [35]. In the case of the neurotensin receptor, the ligand neurotensin was used to construct a recombinant fusion protein with exotoxin A. Binding experiments showed that the recombinant proteins were able to recognize their target structures, and in vitro cytotoxicity assays with neurotensin-ETA showed specific antitumor activity for receptor-positive tumor cell lines. This work led to the conclusion that transmembrane spanning receptors are potential targets, which allow toxin binding and promote tumor cell killing.

\section{Signal Transducers and Activators of Transcription}

Signal transducers and activators of transcription (STATs) are a family of cytoplasmic proteins with roles as signal messengers and transcription factors that participate in normal cellular responses to cytokines and growth factors. Frequently, however, abnormal activity of certain STAT family members, particularly Stat 3 and Stat5, is associated with human malignancies. It was shown that Stat 3 has a causal role in oncogenesis and might be a tar- get for cancer drugs and therapeutic intervention. Furthermore, a constitutively active mutant (Stat3) induces transformation of cells, which form tumors in vivo. Activation of Stat 3 signaling is accompanied by upregulation of cyclin D1, c-Myc, and Bcl-x. The blocking of constitutive Stat 3 signaling results in growth inhibition and apoptosis of Stat3-positive tumor cells in vitro and in vivo. The dependence of certain tumors on constitutive Stat 3 signaling for growth and survival has wide implications for cancer therapy. Therefore various strategies for targeting Stat 3 signaling for therapeutic intervention are being considered [36]. However, at this moment, no synthetic STAT inhibitor is being available for the use in therapy.

As STAT proteins are transcription factors activated by phosphorylation on tyrosine residues after cytokine stimulation, their role in the functioning of various receptors and enzymatic cascades is being investigated. For example, in erythropoietin receptor (EPOR)-mediated signaling. STAT5 is tyrosine-phosphorylated by EPO stimulation. Several studies [37-41] indicate that STAT activation is caused by members of other families of protein tyrosine kinases such as the Src family. In a study by Okutani et al. [37] reduction of Src by induction of antisense Src RNA expression suppressed EPO-promoted erythroid differentiation in K562 cells. In addition the function of Src downstream of the EPOR-initiated signaling was recently investigated, and it was found that reduction of Src diminished tyrosine phosphorylation of STAT5 in K562 cells regardless of EPO treatment. Further, the tyrosine phosphorylation level of STAT5 induced by EPO in F-36P cells was reduced in the presence of PP1- or PP2-selective Src inhibitor. In addition, the expression of dominant negative Src in F-36P cells reduced the tyrosine phosphorylation of STAT5. When Src and STAT5 were coexpressed in COS7 cells, tyrosine phosphorylation of STAT5 was observed. Additionally, a tyrosine residue of STAT5A was already identified as the major phosphorylation site by Scr [37].

The STATs and their functions represent a complex issue. Recent studies have led to the identification of numerous potential targets for cancer therapy, including many proteins that normally function in signal transduction pathways downstream from hematopoietic growth factor receptors. Autophosphorylation of tyrosine residues attracts a variety of adapter proteins and other signaling proteins, leading to cell growth, viability, and adhesion signals of malignant cells. It is now becoming possible to link specific signaling pathways to biological abnormalities, for example in CML cells [38]. In vivo studies of certain lines of transgenic mice already suggest the impor- 
tance of STATs antiapoptotic effects. Clinically important benefits from the discovery of the contribution of STAT activation to oncogenesis include the development of new diagnostic and prognostic assays based on the molecular STAT profile of tumors [39-41]. Furthermore, as STAT activation has been shown to be required for oncogenic transformation, discovery and development of novel inhibitors of STAT signaling might promise new antitumor compounds. Therefore these studies may lead to the discovery of new therapeutic targets and specific therapeutic compounds.

\section{Other Enzymes Related to Protein Phosphorylation}

Many target molecules have been identified for possible use in cancer therapy. These targets are typically components of supramolecular complexes such as transcription factors, nuclear pore proteins, or cytoskeletal components. For example, the enzymes responsible for O-linked $\mathrm{N}$-acetylglucosamine (O-GlcNAc) addition and removal are highly conserved molecules. They play an important role in cell signaling. The O-GlcNac transferase and OGlcNAcase are likely to act in concert with kinases and phosphatases generating various isoforms of physiological substrates. These isoforms may differ in such properties as protein-protein interactions, protein stability, and enzymatic activity. Altered O-GlcNAc metabolism may play a role in cancer [42] and may therefore be used for the purposes of cancer chemotherapy.

\section{Ribonucleotide Reductase}

The enzyme ribonucleotide reductase is responsible for the formation of deoxynucleosidediphosphates from ribonucleotidediphosphates. It is a key enzyme of 'de novo' DNA synthesis. The enzyme activity of ribonucleotide reductase was shown by Elford et al. [43] and Takeda and Weber [44] to be significantly increased in a transformation- and proliferation-linked manner $[43,44]$. In an animal model for tumors with different growth rates (Morris hepatoma) it was shown that malignant cells have significantly higher enzyme activities than normal liver cells [43]. In addition, the enzyme activity significantly increased with the doubling times of various tumors. Therefore, the enzyme is considered to be an excellent target for cancer chemotherapy.

New Developments in Cancer

Chemotherapy
A number of compounds were identified as inhibitors of ribonucleotide reductase. These compounds include hydroxyurea, a drug which is widely used in the treatment of hematological malignancies as well as other specific inhibitors of the enzyme. Newer compounds which inhibit ribonclueotide reductase are polyhydroxy substitutes, benzohydroxamic acid derivatives, difluorodeoxycytidine (Gemcitabine), Fludarabine or thiosemicarbazones. Gemcitabine is being used to treat solid tumors and Fludarabine is effective against hematological malignancies. Thiosemicarbazones are presently being studied and might be promising as new antitumor agents [45-48].

Over the past years our group investigated the effects of various polyhydroxy substitutes of benzohydroxamic acid derivatives [49-51]. These compounds effectively inhibit the growth of various tumor cell lines as well as in vivo tumors as single agents, and in combination with other chemotherapeutic agents, such as adriamycin. They might be a promising additional option for the treatment of various malignancies [49-51].

\section{The Use of Hypoxia-Selective Cytotoxins}

Hypoxic tumor cells have shown high resistance against radiation therapy or chemotherapy and therefore represent an important target for cancer therapy [52-54]. Various compounds have been synthesized to specifically target this tumor cell population in order to avoid the emergence of hypoxic cancer cells from therapy. In particular, solid tumor cells have the tendency to form hypoxic cells; these cells may cause acceleration of malignant progression and metastasis. Several compounds that are only activated to cytotoxic compounds in hypoxic cells have been synthesized. One of these compounds, tirapazamine, is presently in phase III clinical studies for the treatment of hypoxic solid tumors [55, 56]. Tirapazamine is being metabolized to a transient oxidizing radical. Normal tissues can easily scavenge the radical and reform the parent compound. In the absence of oxygen, however, the compound causes DNA stand breaks and therefore was shown to be an effective anticancer drug. In anoxic cells in culture, the compound proved to be approximately 100-fold more potent than under oxic conditions. In addition, the compound showed significant synergy with radiation therapy as well as in combination with other anticancer drugs such as cisplatin [57]. Therefore, this compound might have turned a problem, i.e. treatment of hypoxic tumor cells, into an advantage, especially since various solid tumors, such as lung cancer, that are extremely diffi-

Med Principles Pract 2002;11:117-125 
cult to treat, which might in part be due to the resistance of hypoxic tumor cells. These cells can now be specifically targeted and therefore tirapazamine might become a very important additional drug in the combination treatment of solid tumors.

\section{Conclusion}

Cancer is a disease which is the consequence of viral, genetic, hormonal or nutritional defects that lead to the development of tumors. Therefore, effective prevention and treatment may differ from tumor to tumor and from patient to patient. However, researchers try to identify specific targets for therapy and thereby hope to effectively inhibit tumor progression. We have summarized some important new targets for cancer chemotherapy. We have included a number of new promising targets and have omitted classical enzymatic targets that are well understood and which have been extensively described in the past. Clearly, we have to be aware that targeting one single enzyme or pathway may not be enough for successful chemotherapeutic treatment of patients. Various compounds with different modes of action must be combined in order to achieve an optimal treatment outcome. It is also important to note that even old drugs might have beneficial effects on various unknown targets or pathways. Elucidating these effects might help in the design of new promising regimens and combination strategies.

\section{References}

1 Szyf M: The DNA methylation machinery as a therapeutic target. Curr Drug Targets 2000;1: 101-118.

2 Karpf AR, Moore BC, Ririe TO, Jones DA: Activation of the p53 DNA damage response pathway after inhibition of DNA methyltransferase by 5-aza-2'-deoxycytidine. Mol Pharmacol 2001;59:751-757.

3 Tateno M, Fukunishi Y, Komatsu S, Okazaki Y, Kawai J, Shibata K, Itoh M, Muramatsu M, Held WA, Hayashizaki Y: Identification of a novel member of the snail/Gfi-1 repressor family, mlt 1 , which is methylated and silenced in liver tumors of SV40 T antigen transgenic mice. Cancer Res 2001;61:1144-1153.

4 Yang X, Ferguson AT, Nass SJ, Phillips DL, Butash KA, Wang SM, Herman JG, Davidson NE: Transcriptional activation of estrogen receptor alpha in human breast cancer cells by histone deacetylase inhibition. Cancer Res 2000;60:6890-6894.

5 Finnegan NM, Curtin JF, Prevost G, Morgan B, Cotter TG: Induction of apoptosis in prostate carcinoma cells by $\mathrm{BH} 3$ peptides which inhibit $\mathrm{Bak} / \mathrm{Bcl}-2$ interactions. $\mathrm{Br} \mathrm{J}$ Cancer 2001;85:115-121.

6 Kim SG, Kim SN, Jong HS, Kim NK, Hong SH, Kim SJ, Bang YJ. Caspase-mediated Cdk2 activation is a critical step to execute transforming growth factor-beta-1-induced apoptosis in human gastric cancer cells. Oncogene 2001;20:1254-1265.

7 Howell SB: Resistance to apoptosis in prostate cancer cells. Mol Urol 2000;4:225-229.

8 Henkels KM, Turchi JJ: Cisplatin-induced apoptosis proceeds by caspase-3-dependent and -independent pathways in cisplatin-resistant and -sensitive human ovarian cancer cel lines. Cancer Res 1999;59:3077-3083.
9 Li L, Zhu Z, Joshi B, Porter AT, Tang DG: A novel hydroxamic acid compound. BMD188, demonstrates anti-prostate cancer effects by inducing apoptosis. I. In vitro studies. Anticancer Res 1999; 19:51-60.

10 Martins LM, Kottke TJ, Kaufmann SH, Earnshaw WC: Phosphorylated forms of activated caspases are present in cytosol from HL-60 cells during etoposide-induced apoptosis. Blood 1998;92:3042-3049.

11 Tse E, Rabbits TH: intracellular antibody-caspase-mediated cell killing: An approach for application in cancer therapy. Proc Natl Acad Sci USA 2000;97:12266-12271.

12 Somerville L, Cory JG: Enhanced roscovitineinduced apoptosis is mediated by a caspase3-like activity in deoxyadenosine-resistant mouse leukemia L1210 cells. Anticancer Res 2000;20:3347-3355.

13 Traxler P, Bold G, Buchdunger E, Caravatti G, Furet P, Manley P, O'Reilly T, Wood J, Zimmermann J: Tyrosine kinase inhibitors: From rational design to clinical trials. Med Res Rev 2001;21:499-512.

14 Thant AA, Nawa A, Kikkawa F, Ichigotani Y, Zhang Y, Sein TT, Amin AR, Hamaguchi M: Fibronectin activates matrix metalloproteinase-9 secretion via the MEK1-MAPK and the P13K-Akt pathways in ovarian cancer cells. Clin Exp Metastasis 2000;18:423-428.

15 Longati P, Comoglio PM, Bardelli A: Receptor tyrosine kinases as therapeutic targets: The model of the MET oncogene. Curr Drug Targets 2001;2:41-55.

16 Lee KS, Rha SY, Kim SJ, Kim JH, Roh JK, Kim BS, Chung HC: Sequential activation and production of matrix metalloproteinase-2 during breast cancer progression. Clin Exp Metastasis 1996;14:512-519.
17 Nieves-Neira W, Pommier Y: Apoptotic response to camptothecin and 7-hydroxystaurosporine (UCN-01) in the 8 human breast cancer cell lines of the NCI Anticancer Drug Screen: Multifactorial relationships with topoisomerase I, protein kinase C, Bcl-2, p53, MDM-2 and caspase pathways. Int J Cancer 1999;82:396404.

18 Huang $\mathrm{CH}$, Treat J: New advances in lung cancer chemotherapy: Topotecan and the role of topoisomerase I inhibitors. Oncology 2001; 61(suppl 1):14-24.

19 Johnston SRD, Kelland LR: Farnesyl transferase inhibitors - a novel therapy for breast cancer. Endocr Relat Cancer 2001;8:227-235.

20 Sakowski J, Bohm M, Sattler I, Dahse HM, Schlitzer M: Synthesis, molecular modeling, and structure-activity relationship of benzophenone-based CAAX-peptidomimetic farnesyltransferase inhibitors. J Med Chem 2001;44: 2886-2899.

21 Slamon DJ, Leyland-Jones B, Shal S, Fuchs H, Paton V, Bajamonde A, Fleming T, Eiermann W, Wolter J, Pegram M, Baselga J, Norton L: Use of chemotherapy plus a monoclonal antibody against HER2 for metastatic breast cancer that overexpresses HER2. N Engl J Med 2001;344:783-792.

22 Barbacid M: Ras genes. Annu Rev Biochem 1987;62:779-782

23 McCormack F: Activators and effectors or ras p21 proteins. Curr Opin Genet Dev 1994;4: 71-76.

24 Reiss Y, Goldstein JL, Seabra MC, Casey PJ, Brown MS: Inhibition of purified p21 Ras farnesyl protein transferase by Cys-AAX tetrapeptides. Cell 1990;62:81-88. 
25 Qian Y, Blaskovich MA, Saleem M, Seong C-M, Wathen SP, Hamilton AD, Sebti SM: Design and structural requirements of potent peptidomimetic inhibitors of p21ras farnesyltransferase. J Biol Chem 1994;269:1241012413.

26 Sepp-Lorenzino L, Ma Z, Rands E, Khol NE, Gibbs JB, Rosen NA: A peptidomimetic inhibitor of farnesyl protein transferase blocks the anchorage-dependent and -independent growth of human tumor cell lines. Cancer Res 1995;55:5301-5309.

27 Johnston SRD, Hickish T, Ellis PA, Houston S, Howes AJ, Dowsett M, Kelland L, Palmer P: Clinical activity with the farnesyl transferase inhibitor R115777 in patients with advanced breast cancer - relationship with tumour phenotype. Breast Cancer Res Treat 2000;64: 32(A28).

28 Karp JE, Lancet JE, Kaufmann SH, End DW, Wright JJ, Bol K, Horak I, Tidwell ML, Liesveld J, Kottke TJ, Ange D, Buddharaju L, Gojo I, Highsmith WE, Belly RT, Hohl RJ, Rybak ME, Thibault A, Rosenblatt J: Clinical and biologic activity of the farnesyltransferase inhibitor R115777 in adults with refractory and relapsed acute leukemias. A phase 1 clinical-laboratory correlative trial. Blood 2001;97:33613369.

29 Punt CJ, van Maanen L, Bol CJ, Seifert WF, Wagener DJ: Phase I and pharmacokinetic study of the orally administered farnesyl transferase inhibitor R115777 in patients with advanced solid tumors. Anticancer Drugs 2001; 12:193-197.

30 Johnston SRD: Farnesyl transferase inhibitors: A novel targeted therapy for cancer. Lancet Oncol 2001;2:18-26.

31 Lavelle F, Riou JF, Laoui A, Mailliet P: Telomerase: A therapeutic target for the third millennium? Crit Rev Oncol Hematol 2000;34: 111-126.

32 Vietor M, Winter S, Groscurth P, Naumann U, Weller M: On the significance of telomerase activity in human malignant glioma cells. Eur J Pharmacol 2000;407:27-37.
33 Llovera M, Touraine P, Kelly PA, Goffin V: Involvement of prolactin in breast cancer: Redefining the molecular targets. Exp Gerontol 2000;35:41-51.

34 Goffin V, Touraine P, Pichard C, Bernichtein S, Kelly PA: Should prolactin be reconsidered as a therapeutic target in human breast cancer? Mol Cell Endocrinol 1999;151:79-87.

35 Wick B, Groner B: Evaluation of cell surface antigens as potential targets for recombinant tumor toxins. Cancer Lett 1997;118:161-172.

36 Turkson J, Jove R: STAT proteins: Novel molecular targets for cancer drug discovery. Oncogene 2000;19:6613-6626.

37 Okutani Y, Kitanaka A, Tanaka T, Kamano H, Ohnishi H, Kubota Y, Ishida T, Takahara J: Src directly tyrosine-phosphorylates STAT5 on its activation site and is involved in erythropoietin-induced signaling pathway. Oncogene 2001;20:6643-6650.

38 Griffin JD: Phosphatidyl inositol signaling by BCR/ABL: Opportunities for drug development. Cancer Chemother Pharmacol 2001; 48(suppl 1):S11-S16.

39 Bowman T, Yu H, Sebti S, Dalton W, Jove R: Signal transducers and activators of transcription: Novel targets for anticancer therapeutics. Cancer Control 1999;6:427-435.

40 Lin TS, Mahajan S, Frank DA: STAT signaling in the pathogenesis and treatment of leukemias. Oncogene 2000;19:2496-2504.

41 Bowman T, Garcia R, Turkson J, Jove R: STATs in oncogenesis. Oncogene 2000;19: 2474-2488.

42 Hanover JA: Glycan-dependent signaling: Olinked N-acetylglucosamine. FASEB J 2001; 15:1865-1876.

43 Elford HL, Freese M, Passamani E, Morris JP: Ribonucleotide reductase and cell proliferation. J Biol Chem 1970;245:5228-5233.

44 Takeda E, Weber G: Role of ribonucleotide reductase in the expression of the neoplastic program. Life Sci 1981;28:1007-1014.

45 Cory JG, Carter GL: Drug action on ribonucleotide reductase. Adv Enzyme Regul 1985; 24:385-401

46 Elford HL: Effect of hydroxyurea on ribonucleotide reductase. Biochem Biphys Res Commun 1968:33:129-135.
47 Plunkett W, Huang P, Gandhi V: Metabolism and action of fludarabine phosphate. Semin Oncol 1990;17:3-17.

48 Plunkett W, Huang P, Searcy CE, Gandhi V: Gemcitabine: Preclinical pharmacology and mechanisms of action. Semin Oncol 1996;23: $3-15$.

49 Riet B van't, Kier LB, Elford HL: A structure activity relationship analysis of benzohydroxamic acid inhibitors of ribonucleotide reductase. J Pharm Sci 1980;69:856-857.

50 Fritzer-Szekeres M, Novotny L, Romanova D, Göbl R, Sedlak J, Vachalkova A, Rauko P, Elford HL, Szekeres T: Enhanced effects of adriamycin by combination with a new ribonucleotide reductase inhibitor, trimidox, in murine leukemia. Life Sci 1998;63:545-552.

51 Szekeres T, Fritzer M, Strobl H, Gharehbaghi K, Findenig G, Elford HL, Lhotka C, Schön HJ, Jayaram HN: Synergistic growth inhibitory and differentiating effects of trimidox and tiazofurin in human promyelocytic leukemia HL60 cells. Blood 1994;84:4316-4312.

52 Kennedy KA: Hypoxic cells as specific drug targets for chemotherapy. Anticancer Drug Des 1987;2:181-194.

53 Brown JM, Giaccia AJ: The unique physiology of solid tumors: Opportunities (and problems) for cancer therapy. Cancer Res 1998;58:14081416 .

54 Brown JM: The hypoxic cell: A target for selective cancer therapy. 18th Bruce F. Cain Memorial Award lecture. Cancer Res 1999;59: 5863-5870.

55 Brown JM: SR4233 (tirapazamine): A new anticancer drug exploiting hypoxia in solid tumours. Br J Cancer 1993;67:1163-1170.

56 Denny WA, Wilson WR: Tirapazamine: A bioreductive anticancer drug that exploits tumour hypoxia. Expert Opin Investig Drugs 2000;9:2889-2901.

57 Gatzemeier U, Rodriguez G, Treat J, Miller V, von Roemeling R, Viallet J, Rey A: Tirapazamine-cisplatin: The synergy. Br J Cancer 1998; 77(suppl 4):15-17. 